\title{
МАСС-СПЕКТРОМЕТРИЯ СУПРАМОЛЕКУЛЯРНЫХ КОМПЛЕКСОВ ГЛИЦИРРИЗИНОВОЙ КИСЛОТЫ И СТРЕПТОМИЦИНА
}

\author{
(C) Е.В. Ветрова, А.В. Лекарь, Е.В. Максименко, С.С. Хизриева, А.Ф. Бугаева, Н.И. Борисенко" \\ НИИ фризической и органической химии Южного федерального \\ университета, пр. Стачки, 194/2, Ростов-на-Дону, 344090 (Россия), \\ e-mail: boni@ipoc.rsu.ru
}

Глицирризиновая кислота (ГК), выделенная из корней солодки голой Glycyrrhiza glabra L. и Gl. uralensis F., обладает уникальными комплексообразующими свойствами, которые широко используются в синтезе новых лекарственных форм на основе молекулярных комплексов с фармаконами. В таких гетерогенных комплексах молекулы ГК выступают в роли полидентантных лигандов, образующих с фармаконами супрамолекулярные комплексы различной стехиометрии. Целью данной работы является изучение комплексообразования растительных гликозидов с антимикробным препаратом стрептомицином для создания новых низкодозных фармсубстанций на основе глицирризиновой кислоты. В представленной работе впервые синтезированы и изучены методом УФ-спектроскопии и массспектрометрии с ионизацией электрораспылением гетерогенные супрамолекулярные комплексы ГК с антибиотиком стрептомицином (СТ), полученные при различных концентрациях «хозяина»и «гостя» в соотношении $1: 1$ и $4: 1$. Зарегистрированные изменения в спектре поглощения СТ в присутствии ГК с помощью УФ-спектроскопии доказывают формирование гетерогенных комплексов ГК-СТ в жидкой фазе. С использованием масс-спектрометрии зарегистрировано эффективное взаимодействие ГК и СТ с образованием различных гетерогенных супрамолекулярных структур с соотношением «хозяин: гость» $1: 1,2: 1,3: 1,3: 2$. При увеличении концентрации ГК наблюдали как увеличение степени самоассоциации ГК (до 4), так и образование комплекса с соотношением $4: 1$. Результаты исследований демонстрируют высокий потенциал использования глицирризиновой кислоты для синтеза новых форм антибактериального препарата стрептомицина в виде нековалентных молекулярных комплексов.

Ключевые слова: глицирризиновая кислота, стрептомицин, масс-спектрометрия с ионизацией электрораспылением, супрамолекулярные комплексы.

Работа выполнена при финансовой поддержке внутреннего гранта Южного федерального универсиmema № 213.01-2014/005BГ.

\section{Введение}

Глицирризиновая кислота (ГК) (рис. 1) является основным тритерпеновым гликозидом корней солодки голой Glycyrrhiza glabra L. и Gl. uralensis F. и обладает широким спектром противовирусной и иммунотропной активности $[1,2]$. Уникальные комплексообразующие свойства глицирризиновой кислоты используются в синтезе новых лекарственных форм на основе молекулярных комплексов с фармаконами,

Ветрова Елена Владимировна - кандидат биологических наук, старший научный сотрудник,

e-mail: boni@ipoc.rsu.ru

Лекарь Анна Владимировна - научный сотрудник, e-mail: boni@ipoc.rsu.ru

Максименко Елена Владимировна - научный сотрудник, e-mail: boni@ipoc.rsu.ru

Хизриева Салима Салимовна - магистрант, e-mail: boni@ipoc.rsu.ru

Бугаева Анастасия Федоровна - магистрант, e-mail: boni@ipoc.rsu.ru

Борисенко Николай Иванович - доктор химических наук, главный научный сотрудник, e-mail: boni@ipoc.rsu.ru которые позволяют снизить суточную дозу, сформировать пролонгированное действие исходных лекарственных веществ и сохранить их фармакологическую эффективность [3, 4]. В таких гетерогенных комплексах молекулы ГК выступают в роли полидентантных лигандов, образующих с фармаконами супрамолекулярные комплексы различной стехиометрии [5-7]. Для установления фундаментальных связей структура - свойство важно изучение механизмов образования комплексов «хозяин:

\footnotetext{
* Автор, с которым следует вести переписку.
} 
гость», где ГК выступает в качестве молекулы «хозяина». Ранее авторами с использованием квантовохимических расчетов и масс-спектрометрии показано, что молекулы ГК способны формировать устойчивые самоассоциаты $(\mathrm{Xn}, \mathrm{n}=2-6)$ с образованием эндолипофильной полости, достаточной для включения соразмерных молекул «гостей» [8].

Цель представленной работы - продолжение работ по изучению комплексообразования биологически активных метаболитов с антимикробными препаратами для создания новых низкодозных фармсубстанций на основе глицирризиновой кислоты. В данной работе впервые синтезированы и изучены (методами УФ-спектроскопии и масс-спектрометрии с ионизацией электрораспылением) гетерогенные супрамолекулярные комплексы ГК с широко используемым антибиотиком стрептомицином (СТ), синтезированные при различных соотношениях «хозяин : гость».

Актуальность представленных исследований обусловлена тем, что стрептомицин (рис. 1) эффективен при лечении самых различных заболеваний, вызываемых грамположительными, грамотрицательными и кислотоустойчивыми бактериями. Особенно эффективен стрептомицин к возбудителю туберкулеза Mycobacterium tuberculosis hominis. Но наряду с высокой эффективностью при лечении инфекционных заболеваний использование стрептомицина сопровождается сильными токсическими эффектами, также для антибиотика характерно чрезвычайно быстрое привыкание к нему бактерий и выработка стрептомициноустойчивых форм, что приводит к необходимости повышения доз препарата и усилению негативных эффектов $[9,10]$. Решением данной проблемы может быть создание новой формы антибактериального препарата $[11,12]$ в виде комплексов с глицирризиновой кислотой, что позволит снизить побочные эффекты и увеличить биодоступность препарата.

С химической точки зрения стрептомицин представляет собой сильное органическое основание (рис. 1). Основной характер его действия обусловлен наличием в молекуле трех группировок: двух гуанидиновых остатков и $\mathrm{N}$-метильной группы сахарного компонента молекулы.

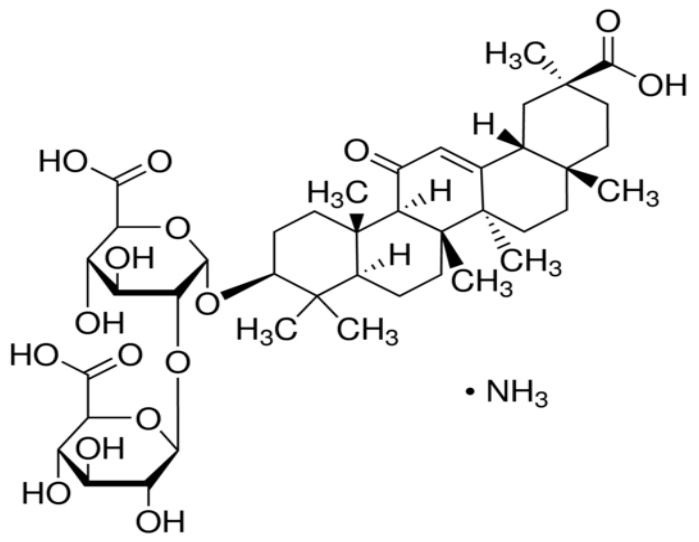

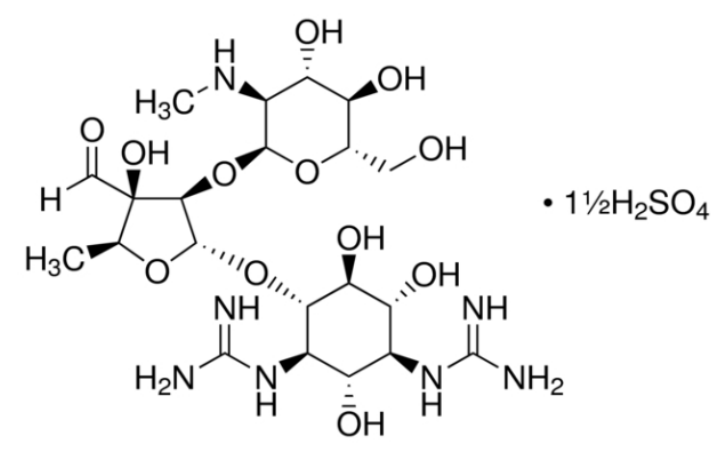

Глицирризиновая кислота (ГК) $\mathrm{C}_{42} \mathrm{H}_{62} \mathrm{O}_{16} \cdot \mathrm{NH}_{3}(m / z=839,4297)$

$$
\begin{gathered}
\text { Стрептомицин (СТ) } \\
\mathrm{C}_{21} \mathrm{H}_{39} \mathrm{~N}_{7} \mathrm{O}_{12} \cdot 1,5 \mathrm{H}_{2} \mathrm{SO}_{4}(m / z=728,2161)
\end{gathered}
$$

Рис. 1. Структурные формулы и моноизотопные массы свободных молекул глицирризиновой кислоты (ГК) и стрептомицина (СТ)

Являясь основанием, стрептомицин легко образует соли как с минеральными, так и с органическими кислотами. Препараты стрептомицина, применяемые в медицине, обычно представляют собой соли стрептомицина с серной (сульфат), хлороводородной (гидрохлорид), фосфорной (фосфат) кислотами и др. При этом соли стрептомицина более устойчивы, чем его основание, и не теряют активности при хранении их в течение нескольких лет.

\section{Экспериментальная часть}

Для исследований использовали моноаммонийную соль ГК производства «Sigma», стрептомицин сульфат (аптечный препарат, производитель ОАО «Биохимик») и растворители фирмы Merck, квалификации HPLC/MS. 
Комплексы в водно-метанольном (20\%) растворе получены путем смешивания растворов ГК и СТ. Полученные смеси в соотношении $1: 1$ (или $4: 1$ ) содержали 0,1 мМ (или 0,4 мМ) ГК и 0,1 мМ СТ.

Комплексообразование ГК с СТ регистрировали с помощью УФ-Вид спектроскопии на спектрофотометре СПЕКС ССП 705 (190-1100 нм) (производитель ЗАО «Спектроскопические системы», РФ). О формировании комплекса судили по изменению оптической плотности стрептомицина (рис. 2). Для исключения вклада поглощения самой ГК ее спектр поглощения вычитался из суммарного спектра. Измерения проводили в кварцевой кювете.

Состав супрамолекулярных комплексов ГК с СТ исследовали при прямом вводе на массспектрометре Bruker Daltonics micrOTOF-Q с ионизацией электрораспылением. Для анализа результатов использовали программное обеспечение micrOTOFcontrol ${ }^{\mathrm{TM}} 2.2$. Калибровка масс-спектрометра выполнена с использованием калибровочного раствора для электрораспыления (Electrospray Calibrant Solution) фирмы Fluka. Детектирование отрицательных и положительных ионов выполнено в интервале $m / z$ от 50 до 3000 . Напряжение на капилляре распылителя \pm 4200 В, параметры газа-осушителя (азот квалификации «о.с.ч.», 5 л/мин, $\left.200^{\circ} \mathrm{C}\right)$ и энергия ионов на квадруполе (5,0 эВ) оптимизированы для детектирования пиков ассоциатных ионов. Чувствительность измерений на micrOTOF-Q II достигает выше 1-2 ppm, точность определения масс - 2 мДа. В данной работе высокая точность измерений не требовалась, поскольку для получения супрамолекулярных комплексов использованы известные исходные химические реактивы, использование которых позволяет однозначно интерпретировать состав регистрируемых ионов. Соотношения $m / z$ и относительные интенсивности пиков ионов (I, \%) приведены в таблицах 1-2.

\section{Результаты и обсуждение}

Комплексы СТ с ГК получены с помощью традиционного смешивания растворов с мольным соотношением компонентов $1: 1$ и $4: 1$. На первом этапе исследований с использованием УФ-спектроскопии изучено формирование комплексов ГК-СТ в растворе. Проведено сравнение спектров поглощения СТ в присутствии ГК и без нее (рис. 2). При добавлении ГК наблюдали гипохромный эффект в спектре поглощения СТ и батохромный сдвиг от $\lambda_{\max }=198$ нм до $\lambda_{\max }=202$ нм. С ростом концентрации ГК в образце наблюдали усиление изменений в спектре поглощения СТ. Зарегистрированные изменения в спектре поглощения СТ в присутствии ГК доказывают формирование гетерогенных комплексов ГК-СТ в жидкой фазе.

Анализ состава полученных комплексов ГК-СТ выполнен с использованием масс-спектрометрии с ионизацией электрораспылением. На первом этапе исследованы масс-спектры положительных и отрицательных ионов СТ, характерные пики зарегистрированных ионов СТ представлены в таблице 1.

В масс-спектрах отрицательных ионов СТ основной пик $m / z$ 678,26 соответствует однозарядному депротонирорванному иону СТ $\left[\mathrm{M}^{\mathrm{CT}} \cdot \mathrm{H}_{2} \mathrm{SO}_{4}-\mathrm{H}\right]^{-}$. СТ в масс-спектре зарегистрирован в виде $\left[\mathrm{M}^{\mathrm{CT}} \cdot 2 \mathrm{H}_{2} \mathrm{SO}_{4}\right.$ -

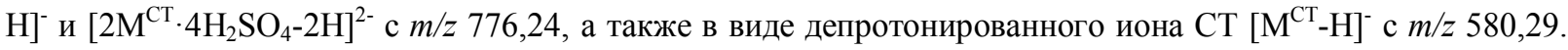
В масс-спектрах зарегистрировано образование самоассоциатов, которые представлены в виде димеров, тримеров и тетрамеров СТ. Одно- и двузарядные депротнированные анионы представлены пиками $m / z$ $1259,54,727,25,1357,53,1017,90$ и 1066,88 интенсивности которых не превышали 2,5\%.

Рис. 2. Спектр поглощения

стрептомицина: 1 - СТ (0,05 мМ);

2 - комплекс с ГК $1: 1$;

3 - комплекс с ГК 4 : 1

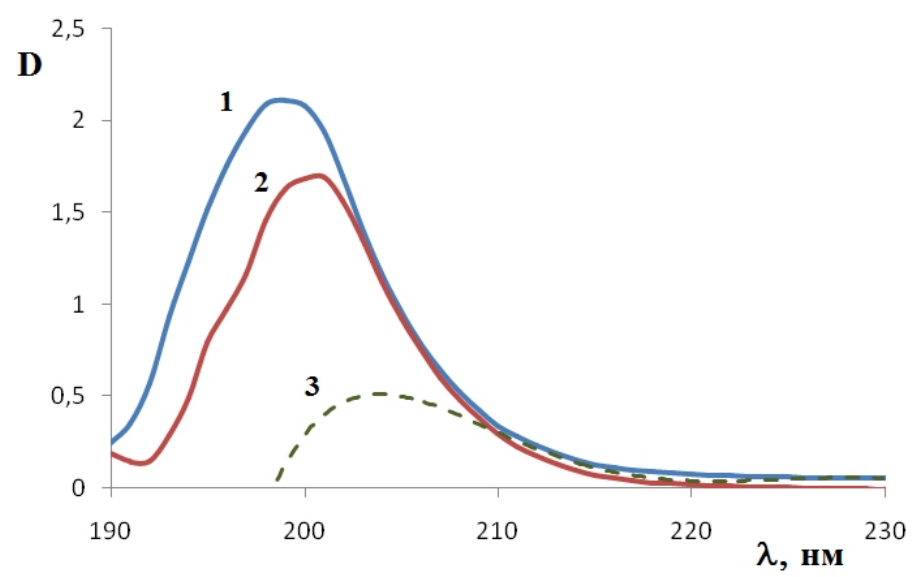


Таблица 1. Характерные ионы для масс-спектров отдельных растворов стрептомицина (CT) и глицирризиновой кислоты (ГК)

\begin{tabular}{|c|c|c|c|}
\hline Состав иона & $\mathrm{m} / \mathrm{z}(\mathrm{I}, \%)$ & Состав иона & $\mathrm{m} / \mathrm{z}(\mathrm{I}, \%)$ \\
\hline$\left[\mathrm{M}^{\mathrm{CT}} \cdot \mathrm{H}_{2} \mathrm{SO}_{4}-\mathrm{H}\right]^{-}$ & $678,26(100)$ & {$\left[\mathrm{M}^{\mathrm{TK}}-2 \mathrm{H}\right]^{2-}$} & $410,20(100)$ \\
\hline$\left[\mathrm{M}^{\mathrm{CT}} \cdot-\mathrm{H}\right]^{-}$ & $580,29(1,21)$ & {$\left[\mathrm{M}^{\Gamma \mathrm{K}}-\mathrm{H}\right]^{1-}$} & $821,41(40,12)$ \\
\hline$\left[\mathrm{M}^{\mathrm{CT}} \cdot 2 \mathrm{H}_{2} \mathrm{SO}_{4}-\mathrm{H}\right]^{-}$и $\left[2 \mathrm{M}^{\mathrm{CT}} \cdot 4 \mathrm{H}_{2} \mathrm{SO}_{4}-2 \mathrm{H}\right]^{2-}$ & $776,24(2,48)$ & {$\left[\mathrm{M}^{\Gamma \mathrm{K}}-3 \mathrm{H}\right]^{3-}$} & $273,13(0,54)$ \\
\hline$\left[2 \mathrm{M}^{\mathrm{CT}} \cdot \mathrm{H}_{2} \mathrm{SO}_{4}-\mathrm{H}\right]^{-}$ & $1259,54(0,32)$ & {$\left[2 \mathrm{M}^{\Gamma \mathrm{K}}-\mathrm{H}\right]^{1-}$ и $\left[4 \mathrm{M}^{\Gamma \mathrm{K}}-2 \mathrm{H}\right]^{2-}$} & $1643,81(7,08)$ \\
\hline$\left[2 \mathrm{M}^{\mathrm{CT}} \cdot 3 \mathrm{H}_{2} \mathrm{SO}_{4}-2 \mathrm{H}\right]^{2-}$ & $727,25(0,56)$ & {$\left[3 \mathrm{M}^{\Gamma \mathrm{K}}-2 \mathrm{H}\right]^{2-}$} & $1232,61(8,22)$ \\
\hline$\left[2 \mathrm{M}^{\mathrm{CT}} \cdot 2 \mathrm{H}_{2} \mathrm{SO}_{4}-\mathrm{H}\right]^{-}$и $\left[4 \mathrm{M}^{\mathrm{S}} \cdot 4 \mathrm{H}_{2} \mathrm{SO}_{4}-2 \mathrm{H}\right]^{2-}$ & $1357,53(1,95)$ & {$\left[\mathrm{M}^{\Gamma \mathrm{K} \cdot \mathrm{NH} 3}-\mathrm{H}\right]^{-}$} & $837,40(11,43)$ \\
\hline$\left[3 \mathrm{M}^{\mathrm{CT}} \cdot 3 \mathrm{H}_{2} \mathrm{SO}_{4}-2 \mathrm{H}\right]^{2-}$ & $1017,90(0,94)$ & {$\left[\mathrm{M}^{\Gamma \mathrm{K} \cdot \mathrm{NH} 3}-2 \mathrm{H}\right]^{2-}$} & $418,16(20,07)$ \\
\hline$\left[3 \mathrm{M}^{\mathrm{CT}} \cdot 4 \mathrm{H}_{2} \mathrm{SO}_{4}-2 \mathrm{H}\right]^{2-}$ & $1066,88(1,51)$ & {$\left[3 \mathrm{M}^{\Gamma \mathrm{K}}+\mathrm{NH}_{3}-2 \mathrm{H}\right]^{2-}$} & $1241,11(2,25)$ \\
\hline$[\mathrm{M}]^{1+}$ & $439,33(100)$ & {$\left[\mathrm{M}^{\Gamma \kappa}+\mathrm{H}\right]^{+}$} & $823,41(100)$ \\
\hline$\left[\mathrm{M}^{\mathrm{CT}} \cdot \mathrm{H}_{2} \mathrm{SO}_{4}+\mathrm{Na}+\mathrm{H}\right]^{2+}$ & $351,70(2,26)$ & {$\left[\mathrm{M}^{\Gamma \mathrm{K}}+\mathrm{NH}_{4}\right]^{+}$} & $840,44(82,17)$ \\
\hline$\left[\mathrm{M}^{\mathrm{CT}} \cdot \mathrm{H}_{2} \mathrm{SO}_{4}+\mathrm{K}+\mathrm{H}\right]^{2+}$ & $359,69(37,40)$ & {$\left[\mathrm{M}^{\Gamma \mathrm{K}}+\mathrm{Na}\right]^{+}$} & $845,40(7,00)$ \\
\hline \multirow[t]{4}{*}[\mathrm{M}^{\mathrm{CT}}\cdot2\mathrm{H}_{2}\mathrm{SO}_{4}+\mathrm{K}+\mathrm{H}]{$^{2+}$} & $408,59(2,59)$ & {$\left[\mathrm{M}^{\Gamma \mathrm{K}}+\mathrm{K}\right]^{+}$} & $861,37(3,68)$ \\
\hline & & {$\left[2 \mathrm{M}^{\Gamma \mathrm{K}}+\mathrm{NH}_{4}\right]^{+}$} & $1662,68(4,29)$ \\
\hline & & {$\left[3 \mathrm{M}^{\Gamma \mathrm{K}}+2 \mathrm{NH}_{4}\right]^{2+}$} & $1251,61(18,35)$ \\
\hline & & {$\left[5 \mathrm{M}^{\Gamma \mathrm{K}}+3 \mathrm{NH}_{4}\right]^{3+}$} & $1388,70(2,19)$ \\
\hline
\end{tabular}

В масс-спектрах положительных ионов СТ представлен пиками двузарядных катионизированных $\mathrm{Na}^{+}$и $\mathrm{K}^{+}$мономеров. Основной пик в масс-спектре положительных ионов $m / z$ 439,33 соответствует однозарядному иону, состав которого в данной работе не идентифицирован. В то же время в масс-спектре отрицательных ионов все основные пики принадлежат депротонированным молекулярным структурам антибиотика и соответствуют сульфатной соли моно-, ди-, три- и тетрамеров СТ.

Образование комплексов с глицирризиновой кислотой также зарегистрировано с использованием масс-спектрометрии (табл. 2). Анализ масс-спектров показал, что помимо пиков, принадлежащих отдельным компонентам ГК и СТ, присутствуют пики, соответствующие разнообразным по составу гетерогенным супрамолекулярным структурам.

В масс-спектрах отрицательных ионов смеси зарегистрированы слабоинтенсивные пики $\mathrm{m} / z$ 678,24 и 776,24. $(<0,5)$, соответствующие свободному СТ. При добавлении ГК в масс-спектрах отсутствуют пики, соответствующие самоассоциатам молекул СТ (ди-, три- и тетрамерам), характерные для чистого раствора СТ (табл. 1).

При исследовании комплексов в масс-спектрах отрицательных ионов основные пики принадлежали

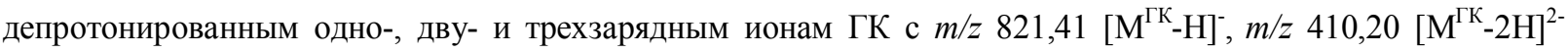
и $m / z 273,13\left[\mathrm{M}^{\Gamma К}-3 \mathrm{H}\right]^{3-}$, как и в случае свободной ГК без СТ (табл. 1). Также в полученном образце зарегистрированы пики $m / z$ 1643,83 и $m / z$ 1232,62, соответствующие самоассоциатам ГК: депротонированным димеру $\left[2 \mathrm{M}^{\Gamma \mathrm{K}}-\mathrm{H}\right]^{-}$и тетрамеру $\left[4 \mathrm{M}^{\Gamma \mathrm{K}}-2 \mathrm{H}\right]^{2-}$, а также тримеру ГК $\left[3 \mathrm{M}^{\Gamma \mathrm{K}}-2 \mathrm{H}\right]^{2-}$.

В масс-спектре положительных ионов основной пик $m / z$ 307,77 соответствует двузарядному иону СТ $\left[\mathrm{M}^{\mathrm{CT}}+2 \mathrm{NH}_{3}\right]^{2+}$. Других пиков, соответствующих свободному СТ и его самоассоциатам в масс-спектрах не зарегистрировано, что, вероятно, обусловлено связыванием СТ молекулами ГК.

В свободном состоянии ГК в масс-спектрах (табл. 1) положительных ионов зарегистрирована в виде аддуктов с $\mathrm{H}^{+}, \mathrm{NH}_{4}^{+}, \mathrm{Na}^{+}, \mathrm{K}^{+}$, которые представлены пиками однозарядных мономеров $\Gamma$ К с $m / z$ 823,41, $840,44,845,40$ и 861,37 соответственно. Наибольшая интенсивность в смеси ГК и СТ принадлежала протонированному иону $\left[\mathrm{M}^{\Gamma \kappa}+\mathrm{H}\right]^{+}$с $m / z$ 823,41. С увеличением концентрации ГК при получении комплексов в образце $4: 1$ наблюдали уменьшение интенсивности пиков для свободных компонентов СТ и увеличение интенсивности пиков для катионизированных ионов ГК и соответствующих геторогенных комплексов с СТ (табл. 2).

Взаимодействие молекул ГК и СТ с образованием гетерогенных супрамолекулярных комплексов в масс-спектрах как отрицательных, так и положительных ионов представлена достаточно широко. Комплексы ГК и СТ в соотношении $1: 1,2: 1$ и $3: 1$ зарегистрированы в виде пиков двузарядных ионов в масс-спектрах отрицательных ионов. В масс-спектрах положительных ионов интенсивности пиков, принадлежащих супрамолекулярным структурам ГК и СТ, существенно выше, чем в комплексах, зарегистрированных в масс-спектрах отрицательных ионов (табл. 2).

Для получения комплексов использовали моноаммонийную соль ГК и сульфат СТ, поэтому в массспектрах зарегистрированы ионы, в состав которых входит как $\mathrm{NH}_{4}^{+}$, так и $\mathrm{HSO}_{4}{ }^{-}$. 
Таблица 2. Характерные пики в масс-спектрах положительных и отрицательных ионов смеси ГК и СТ

\begin{tabular}{|c|c|c|c|}
\hline $\mathrm{m} / \mathrm{z}$ & Состав иона & $\mathrm{I} \% 1: 1$ & $\mathrm{I} \% 4: 1$ \\
\hline 410,20 & {$\left[\mathrm{M}^{\mathrm{TK}}-2 \mathrm{H}\right]^{2-}$} & 100 & 100 \\
\hline 821,41 & {$\left[\mathrm{M}^{\Gamma \mathrm{K}}-\mathrm{H}\right]^{1-}$} & 40,62 & 38,89 \\
\hline 273,13 & {$\left[\mathrm{M}^{\Gamma \mathrm{K}}-3 \mathrm{H}\right]^{3-}$} & 1,52 & 2,12 \\
\hline 1643,81 & {$\left[2 \mathrm{M}^{\Gamma \mathrm{K}}-\mathrm{H}\right]^{1-}$ и $\left[4 \mathrm{M}^{\Gamma К}-2 \mathrm{H}\right]^{2-}$} & 0,05 & 0,04 \\
\hline 1232,61 & {$\left[3 \mathrm{M}^{\Gamma \mathrm{K}}-2 \mathrm{H}\right]^{2-}$} & 0,10 & 0,17 \\
\hline 837,40 & {$\left[\mathrm{M}^{\Gamma \mathrm{K} \cdot \mathrm{NH} 3}-\mathrm{H}\right]^{-}$} & 1,25 & 0,46 \\
\hline 418,16 & {$\left[\mathrm{M}^{\Gamma \mathrm{K} \cdot \mathrm{NH} 3}-2 \mathrm{H}\right]^{2-}$} & 5,89 & 4,21 \\
\hline 1241,11 & {$\left[3 \mathrm{M}^{\Gamma \mathrm{K}}+\mathrm{NH}_{3}-2 \mathrm{H}\right]^{2-}$} & - & 0,06 \\
\hline 919,37 & {$\left[\mathrm{M}^{\Gamma \mathrm{K}} \cdot \mathrm{H}_{2} \mathrm{SO}_{4}-\mathrm{H}\right]^{-}$} & 0,52 & 0,15 \\
\hline 678,24 & {$\left[\mathrm{M}^{\mathrm{CT}} \cdot \mathrm{H}_{2} \mathrm{SO}_{4}-\mathrm{H}\right]^{-}$} & 0,19 & 0,02 \\
\hline 776,24 & {$\left[\mathrm{M}^{\mathrm{CT}} \cdot 2 \mathrm{H}_{2} \mathrm{SO}_{4}-\mathrm{H}\right]^{-}$и $\left[2 \mathrm{M}^{\mathrm{CT}} \cdot 4 \mathrm{H}_{2} \mathrm{SO}_{4}-2 \mathrm{H}\right]^{2-}$} & 0,03 & - \\
\hline 700,85 & {$\left[\mathrm{M}^{\Gamma \mathrm{K}}+\mathrm{M}^{\mathrm{CT}}-2 \mathrm{H}\right]^{2-}$} & 0,08 & 0,02 \\
\hline 749,82 & {$\left[\mathrm{M}^{\Gamma \mathrm{K}}+\mathrm{M}^{\mathrm{CT}} \cdot \mathrm{H}_{2} \mathrm{SO}_{4}-2 \mathrm{H}\right]^{2-}$} & 0,01 & - \\
\hline 1161,06 & {$\left[2 \mathrm{M}^{\Gamma \mathrm{K}}+\mathrm{M}^{\mathrm{CT}} \cdot \mathrm{H}_{2} \mathrm{SO}_{4}-2 \mathrm{H}\right]^{2-}$} & 0,02 & - \\
\hline 1112,05 & {$\left[2 \mathrm{M}^{\Gamma \mathrm{K}}+\mathrm{M}^{\mathrm{CT}}-2 \mathrm{H}\right]^{2-}$} & 0,07 & 0,03 \\
\hline 1523,30 & {$\left[3 \mathrm{M}^{\Gamma \mathrm{K}}+\mathrm{M}^{\mathrm{CT}} 2 \mathrm{H}\right]^{2-}$} & 0,02 & 0,02 \\
\hline 1539,23 & {$\left[3 \mathrm{M}^{\Gamma \mathrm{K}}+\mathrm{M}^{\mathrm{CT}}+2 \mathrm{NH}_{3}-2 \mathrm{H}\right]^{2-}$} & 0,11 & 0,07 \\
\hline 307,77 & {$\left[\mathrm{M}^{\mathrm{CT}}+2 \mathrm{NH}_{3}\right]^{2+}$} & 100 & 100 \\
\hline 823,41 & {$\left[\mathrm{M}^{\Gamma \mathrm{K}}+\mathrm{H}\right]^{+}$} & 0,16 & 27,15 \\
\hline 840,44 & {$\left[\mathrm{M}^{\Gamma \mathrm{K}}+\mathrm{NH}_{4}\right]^{+}$} & - & 10,91 \\
\hline 845,40 & {$\left[\mathrm{M}^{\Gamma \mathrm{K}}+\mathrm{Na}\right]^{+}$} & 0,13 & 3,00 \\
\hline 861,37 & {$\left[\mathrm{M}^{\Gamma \mathrm{K}}+\mathrm{K}\right]^{+}$} & 0,16 & 1,27 \\
\hline 439,33 & {$[\mathrm{M}]^{1+}$} & 36,70 & 44,36 \\
\hline 703,10 & {$\left[\mathrm{M}^{\Gamma \mathrm{K}}+\mathrm{M}^{\mathrm{CT}}+2 \mathrm{H}\right]^{2+}$} & 4,13 & 9,28 \\
\hline 712,11 & {$\left[\mathrm{M}^{\Gamma \mathrm{K}}+\mathrm{M}^{\mathrm{CT}}+\mathrm{NH}_{3}+2 \mathrm{H}\right]^{2+}$} & 4,35 & 8,41 \\
\hline 719,11 & {$\left[\mathrm{M}^{\Gamma \mathrm{K}}+\mathrm{M}^{\mathrm{CT}}+2 \mathrm{NH}_{3}+2 \mathrm{H}\right]^{2+}$} & 52,17 & 95,07 \\
\hline 727,12 & {$\left[\mathrm{M}^{\Gamma \mathrm{K}}+\mathrm{M}^{\mathrm{CT}}+3 \mathrm{NH}_{3}+2 \mathrm{H}\right]^{2+}$} & 1,68 & - \\
\hline 1114,46 & {$\left[2 \mathrm{M}^{\Gamma \mathrm{K}}+\mathrm{M}^{\mathrm{CT}}+2 \mathrm{H}\right]^{2+}$} & 0,40 & 1,56 \\
\hline 1123,46 & {$\left[2 \mathrm{M}^{\Gamma \mathrm{K}}+\mathrm{M}^{\mathrm{CT}}+\mathrm{NH}_{4}+2 \mathrm{H}\right]^{2+}$} & 0,28 & 1,25 \\
\hline 1130,46 & {$\left[2 \mathrm{M}^{\Gamma \mathrm{K}}+\mathrm{M}^{\mathrm{CT}}+2 \mathrm{NH}_{4}+2 \mathrm{H}\right]^{2+}$} & 1,88 & 11,43 \\
\hline 1071,51 & {$\left[3 \mathrm{M}^{\Gamma \mathrm{K}}+\mathrm{M}^{\mathrm{CT}}+3 \mathrm{H}\right]^{3+}$} & - & 0,29 \\
\hline 1022,53 & {$\left[3 \mathrm{M}^{\Gamma \mathrm{K}}+\mathrm{M}^{\mathrm{CT}}+\mathrm{NH}_{3}+3 \mathrm{H}\right]^{3+}$} & - & 1,26 \\
\hline 1028,51 & {$\left[3 \mathrm{M}^{\Gamma \mathrm{K}}+\mathrm{M}^{\mathrm{CT}}+2 \mathrm{NH}_{3}+3 \mathrm{H}\right]^{3+}$} & 0,16 & 4,97 \\
\hline 1228,03 & {$\left[3 \mathrm{M}^{\Gamma \mathrm{K}}+2 \mathrm{M}^{\mathrm{CT}}+3 \mathrm{NH}_{4}\right]^{3+}$} & 0,38 & 0,49 \\
\hline 1232,95 & {$\left[3 \mathrm{M}^{\Gamma \mathrm{K}}+2 \mathrm{M}^{\mathrm{CT}}+4 \mathrm{NH}_{4}\right]^{3+}$} & 0,61 & 1,04 \\
\hline 1296,43 & {$\left[4 \mathrm{M}^{\Gamma \mathrm{K}}+\mathrm{M}^{\mathrm{CT}}+\mathrm{NH}_{3}+3 \mathrm{H}\right]^{3+}$} & - & 0,62 \\
\hline 1302,41 & {$\left[4 \mathrm{M}^{\Gamma \mathrm{K}}+\mathrm{M}^{\mathrm{CT}}+2 \mathrm{NH}_{3}+3 \mathrm{H}\right]^{3+}$} & - & 2,65 \\
\hline 1307,78 & {$\left[4 \mathrm{M}^{\Gamma \mathrm{K}}+\mathrm{M}^{\mathrm{CT}}+3 \mathrm{NH}_{3}+3 \mathrm{H}\right]^{3+}$} & - & 0,60 \\
\hline
\end{tabular}

В масс-спектрах положительных ионов зарегистрированы пики, соответствующие гетерогенным супрамолекулярным структурам ГК-СТ с соотношением «хозяин - гость» $1: 1,2: 1,3: 1,3: 2$, а при увеличении в образце концентрации ГК повышается степень самоассоциации ГК и регистрируются пики соответствующих гетерогенных комплексов с соотношением $4: 1$.

Наибольшая интенсивность пиков зарегистрирована для комплексов $1: 1$. С наибольшей вероятностью происходит образование комплекса $\left[\mathrm{M}^{\Gamma \mathrm{K}}+\mathrm{M}^{\mathrm{CT}}+2 \mathrm{NH}_{3}+2 \mathrm{H}\right]^{2+}$, для него зарегистрирована наибольшая интенсивность пика $m / z$ 719,11 ( 52\%). В образце с мольным соотношением $4: 1$ интенсивность пика $m / z$ 719,11 достигала 95\%.

Bсе зарегистрированные в масс-спектрах положительных ионов комплексы представлены в виде моноаммонийной соли и представлены пиками дву- и трехзарядных ионов. Наибольшая интенсивность пиков зарегистрирована для комплексов $1: 1,2: 1,3: 1,3: 2$ и $4: 1$, в составе которых присутствует два моноаммонийных остатка $m / z 719,11, m / z 1130,46\left[2 \mathrm{M}^{\Gamma \mathrm{K}}+\mathrm{M}^{\mathrm{CT}}+2 \mathrm{NH}_{4}+2 \mathrm{H}\right]^{2+}, m / z 1028,51\left[3 \mathrm{M}^{\Gamma \mathrm{K}}+\mathrm{M}^{\mathrm{CT}}+2 \mathrm{NH}_{3}\right.$ $+3 \mathrm{H}]^{3+}, m / z 1302,41\left[4 \mathrm{M}^{\Gamma \mathrm{K}}+\mathrm{M}^{\mathrm{CT}}+2 \mathrm{NH}_{3}+3 \mathrm{H}\right]^{3+}$, которые в большей степени стабилизируют супрамолекулярную структуру.

В целом, результаты исследований показывают эффективное образование гетерогенных комплексов ГК с СТ. Способность природного гликозида ГК образовывать самоассоциаты, построенные по типу «голова - хвост», подтверждена ранее многочисленными исследованиями $[8,13,14]$, в том числе квантовохимическими расчетами и масс-спектрометрией. В данной работе установлено, что не только мономер, но и самоассоциаты ГК достаточно эффективно образуют гетерогенные супрамолекулярные комплексы с антибиотиком СТ. 


\section{Заключение}

Таким образом, в представленной работе впервые синтезированы и изучены супрамолекулярные комплексы ГК с широко используемым антимикробным препаратом стрептомицином, полученные при различных концентрациях «хозяина» и «гостя» в соотношении 1:1 и 4:1. С использованием массспектрометрии зарегистрировано эффективное взаимодействие ГК и СТ с образованием различных гетерогенных супрамолекулярных структур с соотношением «хозяин : гость» $1: 1,2: 1,3: 1,3: 2$. С ростом концентрации ГК в образце увеличивается не только эффективность взаимодействия ГК и СТ, но и регистрируется образование дополнительного комплекса с соотношением $4: 1$. Зарегистрированные изменения в спектре поглощения СТ в присутствие ГК с помощью УФ-спектроскопии доказывают формирование гетерогенных комплексов ГК-СТ в жидкой фазе.

Результаты исследований демонстрируют высокий потенциал глицирризиновой кислоты в создании новых форм антибактериального препарата стрептомицина в виде нековалентных молекулярных комплексов.

\section{Список литературь}

1. Tolstikov G.A., Shultz E.E., Baltina L.A., Pokrovsky A.G. Glycyrrhizic acid // Russian Journal of Bioorganic Chemistry. 1997. Vol. 23. N9. Pp. 625-642.

2. Shibata S.A. Drug over the millennia: Pharmacognosy, chemistry, and pharmacology of licorice // Journal Yakugaku Zasshi. 2000. Vol. 120. N10. Pp. 849-862.

3. Baltina L.A., Kondratenko. R.M., Baltina Jr., Plyasunova O.A., Pokrovskii A.G., Tolstikov G.A. Search for new drugs: Prospects for the creation of new antiviral drugs based on glycyrrhizic acid and its derivatives // Pharmaceutical Chemistry Journal. 2009. Vol. 43. N10. Pp. 539-548.

4. Kondratenko R.M., Baltina L.A., Mustafina S.R., Ismagilova A.F., Zarudii F.S., Davydova V.A., Bazekin G.V., Suleimanova G.F., Tolstikov G.A. Complex compounds of glycyrrhizic acid with antimicrobial drugs // Pharmaceutical Chemistry Journal. 2003. Vol. 37. N9. Pp. 485-488.

5. Борисенко С.Н., Ветрова Е.В., Лекарь А.В., Филонова О.В., Борисенко Н.И. Масс-спектрометрия супрамолекулярных комплексов глицирретиновой кислоты с антибиотиком стрептоцидом // Химия растительного сырья. 2015. №3. С. 127-134.

6. Vetrova E.V., Lekar A.V., Filonova O.V., Borisenko S.N., Maksimenko E.V., Borisenko N.I. Study of molecular complexation of glycyrrhizic acid with chloramphenicol by electrospray ionization mass spectrometry // Journal of Natural Science, Biology and Medicine. 2015. Vol. 6. N1. Pp. 40-43.

7. Borisenko S.N., Lekar A.V., Vetrova E.V., Borisenko N.I. Mass spectrometry of supramolecular complexes of glycyrrhizic acid and benzimidazole derivatives // Chemistry of Natural Compounds. 2013. Vol. 49. N5. Pp. 969-971.

8. Борисенко С.Н., Лекарь А.В., Милов А. А., Ветрова Е.В., Борисенко Н.И. Масс-спектрометрия и квантовохимическое исследование процессов самоассоциации молекул глицирризиновой кислоты // Химия растительного сырья. 2013. №2. С. 85-92.

9. Wrzesniok D., Beberok A., Otreba M., Buszman E. Effect of streptomycin on melanogenesis and antioxidant status in melanocytes // Mol Cell Biochem. 2013. Vol. 383. Pp. 77-84.

10. Nurkeeva Z.S., Khutoryanskiy V.V., Mun G.A., Sherbakova M.V., Ivaschenko A.T., Aitkhozhina N.A. Polycomplexes of poly(acrylic acid) with streptomycin sulfate and their antibacterial activity // European Journal of Pharmaceutics and Biopharmaceutics. 2004. Vol. 57. Pp. 245-249.

11. Silva W.F., Cecílio S.G., Magalhães C. LB, Ferreira J. MS, Tótola A.H., de Magalhaes J.C. Combination of extracts from Aristolochia. Cymbifera with streptomycin as a potential antibacterial drug // SpringerPlus. 2013. Vol. 2. N430. Pp. 1-7.

12. Kooti M., Gharineh S., Mehrkhah M., Shaker A., Motamedi H. Preparation and antibacterial activity of $\mathrm{CoFe}_{2} \mathrm{O}_{4} / \mathrm{SiO}_{2} / \mathrm{Ag}$ composite impregnated with streptomycin // Chemical Engineering Journal. 2015. Vol. 259 . N1. Pp. 34-42.

13. Душкин А.Б., Метелева Е.С., Толстикова Т.Г., Хвостов М.В., Долгих М.П., Толстиков Г.А. Комплексирование фармаконов с глицирризиновой кислотой - путь создания препаратов повышенной эффективности // Химия в интересах устойчивого развития. 2010. №18. С. 517-525.

14. Толстикова Т.Г., Толстиков А.Г., Толстиков Г.А. На пути к низкодозным лекарствам // Вестник Российской академии наук. 2007. Т. 77, №10. С. 867-874.

Поступило в редакцию 16 февраля 2016 г. 
Vetrova E.V., Lekar' A.V., Maksimenko E.V., Khizrieva S.S., Bugaeva A.F., Borisenko N.I.* MASS SPECTROMETRY SUPRAMOLECULAR COMPLEXES GLYCYRRHIZIC ACID, AND STREPTOMYCIN

Institute of Physical and Organic Chemistry, Southern Federal University, pr. Stachki, 194/2, Rostov-on-Don, 344090 (Russia),e-mail: boni@ipoc.rsu.ru

Glycyrrhizic acid (HA) isolated from the roots of licorice Glycyrrhiza glabra L. and Gl. uralensis F. has unique complexing properties, which are widely used in the synthesis of new dosage forms on the basis of molecular complexes with Pharmakon. In such heterogeneous complexes HA molecules act as ligands polidentantnyh forming a supramolecular Pharmakon com-plexes with different stoichiometry. The aim of this work is to study the complexation of plant glycosides with antimicrobials streptomycin to create new low-dose pharmaceutical substances on the basis of glycyrrhizic acid. In this study first synthesized and studied by UV-spectroscopy and mass spectrometry with electrospray ionization heterogeneous supramolecular complexes HA antibiotic streptomycin (ST) obtained at different concentrations of "host" and "guest" in the ratio $1: 1$ and $4: 1$. registered changes in the absorption spectrum of CT in the presence of HA by UV spectroscopy proves the formation of heterogeneous ST HA-complexes in the liquid phase. Using mass spectrometry registered effective interaction of HA and PT various heterogeneous form supramolecular structures with an aspect ratio "host : guest" $1: 1,2: 1,3: 1,3: 2$. By increasing HA concentration was observed as an increase in the degree of self-association of HA (up to 4) and complex formation with a ratio $4: 1$. The results of studies show a high potential for the use of glycyrrhizic acid synthesis of new antibiotic streptomycin forms a noncovalent molecular complexes.

Keywords: glycyrrhizin acid, streptomycin, mass spectrometry with electrospray ionization, supramolecular complexes.

\section{References}

1. Tolstikov G.A., Shultz E.E., Baltina L.A., Pokrovsky A.G. Russian Journal of Bioorganic Chemistry, 1997, vol. 23, no. 9, pp. 625-642.

2. Shibata S.A. Journal Yakugaku Zasshi, 2000, vol. 120, no. 10, pp. 849-862.

3. Baltina L.A., Kondratenko. R.M., Baltina Jr., Plyasunova O.A., Pokrovskii A.G., Tolstikov G.A. Pharmaceutical Chemistry Journal, 2009, vol. 43, no. 10, pp. 539-548.

4. Kondratenko R.M., Baltina L.A., Mustafina S.R., Ismagilova A.F., Zarudii F.S., Davydova V.A., Bazekin G.V., Suleimanova G.F., Tolstikov G.A. Pharmaceutical Chemistry Journal, 2003, vol. 37, no. 9, pp. 485-488.

5. Borisenko S.N., Vetrova E.V., Lekar' A.V., Filonova O.V., Borisenko N.I. Khimiia rastitel'nogo syr'ia, 2015, no. 3, pp. 127-134. (in Russ.).

6. Vetrova E.V., Lekar A.V., Filonova O.V., Borisenko S.N., Maksimenko E.V., Borisenko N.I. Journal of Natural Science, Biology and Medicine, 2015, vol. 6, no. 1, pp. 40-43.

7. Borisenko S.N., Lekar A.V., Vetrova E.V., Borisenko N.I. Chemistry of Natural Compounds, 2013, vol. 49, no. 5, pp. 969-971.

8. Borisenko S.N., Lekar' A.V., Milov A. A., Vetrova E.V., Borisenko N.I. Khimiia rastitel'nogo syr'ia, 2013, no. 2, pp. 85-92. (in Russ.).

9. Wrzesniok D., Beberok A., Otreba M., Buszman E. Mol Cell Biochem., 2013, vol. 383, pp. 77-84.

10. Nurkeeva Z.S., Khutoryanskiy V.V., Mun G.A., Sherbakova M.V., Ivaschenko A.T., Aitkhozhina N.A. European Journal of Pharmaceutics and Biopharmaceutics, 2004, vol. 57, pp. 245-249.

11. Silva W.F., Cecílio S.G., Magalhães C. LB, Ferreira J. MS, Tótola A.H., de Magalhaes J.C. SpringerPlus, 2013, vol. 2, no. 430, pp. 1-7.

12. Kooti M., Gharineh S., Mehrkhah M., Shaker A., Motamedi H. Chemical Engineering Journal, 2015, vol. 259, no. 1, pp. 34-42.

13. Dushkin A.B., Meteleva E.S., Tolstikova T.G., Khvostov M.V., Dolgikh M.P., Tolstikov G.A. Khimiia v interesakh ustoichivogo razvitiia, 2010, no. 18, pp. 517-525. (in Russ.).

14. Tolstikova T.G., Tolstikov A.G., Tolstikov G.A. Vestnik Rossiiskoi akademii nauk, 2007, vol. 77, no. 10, pp. 867874. (in Russ.).

Received February 16, 2016

Revised April 1, 2016

\footnotetext{
* Corresponding author.
} 
